\title{
Designing active Maths for undergraduate STEAM students
}

\author{
Domenico Brunetto ${ }^{1}$, Ana Moura Santos ${ }^{2}$
}

${ }^{1}$ Department of Mathematics, Politecnico di Milano, Italy, ${ }^{2}$ Department of Mathematics, Instituto Superior Técnico, University of Lisbon, Portugal.

\begin{abstract}
This work presents a set of student-centred activities that may help undergraduate students understand mathematics in their first year of a STEAM degree. In particular, the authors refer to the difficulties students meet in making connections between syntactic and semantic dimensions in learning mathematics, especially in Linear Algebra topics. The specific goal of this paper is to present and discuss how it can work in the case of linear transformations. This topic stands in the middle of every Linear Algebra standard course and is pivotal in many recent applications, such as computer graphics. The study describes the teaching-learning experience and reports the results of the first pilot study, which involves about 100 undergraduate Architecture students of Politecnico di Milano. One of the peculiarities of this work is its context since the class is composed of heterogeneous group of students, in terms of knowledge background and attitudes towards mathematics. The main findings of this paper are underlining how a studentcentred strategy, based on asynchronous activities and synchronous class discussion, allows misconceptions to emerge and be appropriately addressed.
\end{abstract}

Keywords: Student-centred activities; open educational resources; linear algebra syllabus; linear transformations; architecture students. 


\section{Introduction}

Many scholars show that in Science, Technology, Engineering, Architecture and Maths (STEAM) programs first-year students face difficulties in Maths (Gueudet, 2008; Andrà, et al., 2019), in particular with Linear Algebra (Moura Santos \& Costa, 2020). Gueudet (2008) argued that such difficulties are due to the difference between procedural mathematics at high school and conceptual at university. In Linear Algebra (LA) for instance the concepts are as important as the computations, a concept such as Linear Transformation (LT) can be more easily apprehended when "seen" from a geometrical point of view (Lay et al., 2016), but the high school syllabus is almost everywhere reduced to Calculus (Santiago et al., 2012).

Open Educational Resources (OERs) have proved to be a good learning support in several areas of Higher Education, in particular in STEAM areas. Technological tools freely accessed on the Internet, such as videos, applets and interactive quizzes, can be used inside and outside the classroom, in order to place the student in the central role of the learning process (Prince, 2004). The strategies can vary from pausing a lecture to propose a quick exercise or quiz to completely changing the class dynamics. One of the advantages of applying student-centred (SC) strategies is, for instance, better academic performance (Freeman et al., 2014).

In this article, authors seek to describe the use of several OERs, in an Elementary Mathematics course aimed for a group of Architecture undergraduates in Politecnico di Milano (https://www.polimi.it), with an average attendance of 100 students per semester. The syllabus of the course is divided in two parts, being one dedicated to the fundamentals of Linear Algebra and the other part dedicated to Calculus with one variable. This paper focuses on using OERs for approaching the concept of a LT, as one of the main concepts in Linear Algebra that allows us to interpret many relevant computer applications (Lay et al., 2016).

The presentation unfolds as follows. Firstly, the relevant aspects of the LTs are recalled. Followed by a description of how the instructor designed and carried out a student-centred activity (SCA) in a first-year mathematics course. Finally, a brief discussion on the main findings and the limitations of our proposal closes the work.

\section{Mathematical content: Linear transformations}

Gilbert Strang in the popular essay "Too Much Calculus" highlights "the central role of linear algebra, [whose] applications touch many more students than calculus. [Because] we are in a digital world now." In the middle (or better, in any place) of a LA syllabus stands the concept of a Linear Transformation (LT). It can be treated as one more "key abstraction" of the syllabus or provide a dynamic and graphical representation of a matrix-vector

\footnotetext{
${ }^{1}$ http://www-math.mit.edu/ gs/papers/essay.pdf
} 
multiplication (Lay et al., 2016). Here, for the aforementioned audience, the last approach was adopted. Within LA course, first-year students can encounter simultaneously the most abstract topics in mathematics and the most relevant applications of today's world: the general concepts of vector space, LTs, factorization coexist along with their applicability in computer simulations for fluid-dynamics, and for computer graphics. Teaching/learning this subject can be challenging in many programs due to the abstract character and the formalism of mathematics that students have not been exposed to in school before (Lalaude-Labayle et al., 2018) so often students feel that Maths is "out-of-reach" (Stewart, 2017).

In our context, a heterogeneous Architectural first-year students group, the LA official syllabus only considers elementary ideas for analysing and solving linear systems and their geometrical interpretations. Introducing LT as a result of a matrix operation on vectors in the Euclidean plane $\left(R^{2}\right)$ and in Euclidean space $\left(R^{3}\right)$ allows students to have a geometric flavour of the abstract concept and, at the same time, the basics of computer graphics. This paper focuses on treating LT as a means to figure out the connections between linear systems, matrix-vector multiplication, the graphical view of actions on vectors, and other properties.

The standard definition of a LT is a function $T$ between two vector spaces, for instance acting from $R^{n}$ to $R^{m}$, such that $T(a \underline{u}+b \underline{v})=a T(\underline{u})+b T(\underline{v})$, where $\underline{u}$ and $\underline{v}$ are any vectors in $R^{n}$ and $a$ and $b$ are any real numbers ${ }^{2}$. Moreover, any matrix multiplication is a LT and vice-versa in $R^{n}$, namely each LT $T$ in $R^{n}$ can be represented by a $n \times n$ matrix $A$ resorting to the row-column product, that is $T(\underline{v})=A \underline{v}$. Geometrically and dynamically speaking, multiplications by $2 \times 2$ matrices are LTs in $R^{2}$ that represent reflections, shears, contractions and expansions, rotations and projections. The beauty of the strong connection between LTs, matrix multiplication and geometrical actions is that it will be easier to make sense of compositions of LTs and inverses (Lay et al., 2016).

\section{Methodology}

For this paper, the data have been collected during the mathematics classes and the students' activities in LA and in particular on LTs. Table 1 shows the course schedule and the moments when the data were collected: as a rule, the students attended on Fridays 3 hrs of lectures delivered online, and $3 \mathrm{hrs}$ of tutoring/exercises delivered in a mixed format (half of the students were in presence and half were online); apart for the first two weeks and the last one. During weeks 1 and 2, the students filled in anonymous questionnaires (Q0 and Q1), delivered through Socrative ${ }^{3}$. The answers to Q0 and Q1 allowed us to learn about the gender

\footnotetext{
${ }^{2}$ One can simply think in the special case of $\mathrm{a}=\mathrm{b}=1$ as the vector sum of two vectors, and try to visualize what the definition of LT says in this case: the LT of the vertex $u+v$ of the parallelogram whose other vertices are $u, 0$ and $v$ is equal to the vertex $T(u)+T(v)$ of the parallelogram whose other vertices are $T(u), 0$ and $T(v)$.

${ }^{3}$ Socrative is an educational platform which enables instant feedback polls for a large classroom https://www.socrative.com
} 
and high school provenience of the students, and also to figure out which are their attitudes towards Maths and their study habits. The information provided by these questionnaires, which is shown in the next section, facilitated the instructors' choices of the learning activities to be performed. The LT activities were delivered in week 4 and 5, and are described in detail below, in Section 4. Moreover, as complementary, yet relevant data, the authors report on the students' feedback in a questionnaire (Q3) about the lessons of weeks 4 and 5, and the students' performance at the midterm exam delivered online in week 7 .

Table 1. Course calendar and data collection.

The underlined sessions point out the LT exposition and activities.

\begin{tabular}{|c|c|c|c|c|c|c|}
\hline \multicolumn{7}{|c|}{ October-November 2020} \\
\hline Week 1 & Week 2 & Week 3 & Week 4 & Week 5 & Week 6 & Week 7 \\
\hline Lecture & 2 Lectures & $\begin{array}{l}\text { Tutoring } \\
+ \text { Lecture }\end{array}$ & $\begin{array}{l}\text { Tutoring + } \\
\text { Homework } \\
+ \text { Lecture }\end{array}$ & $\begin{array}{l}\text { Tutoring } \\
+ \text { Lecture }\end{array}$ & $\begin{array}{l}\text { Tutoring } \\
+ \text { Lecture }\end{array}$ & $\begin{array}{l}\text { Tutoring + } \\
\text { Midterm } \\
\text { exam }\end{array}$ \\
\hline Q0 & Q1 & \multicolumn{3}{|c|}{ Linear Transformations } & Q3 & Midterm \\
\hline
\end{tabular}

Source: by authors.

\subsection{Description of the sample}

The total number of students enrolled in the Elementary Mathematics course were 174, among them 100 are first-year students, while 74 are repeaters, i.e., students enrolled in the second or third year of the corresponding Degree, but who still did not pass the Maths exams. From the total number of enrolees, $65 \%$ are female, $47.3 \%$ have a weak mathematical background according to their high school of provenience (ART and Other), while the remaining $52.7 \%$ of the students have a strong background (LS+TE) ${ }^{4}$.

In questionnaire Q1, one question is related to the students' attitudes towards mathematics To what extent do you feel confident in your performance for this mathematics course? Only 76 students answered the question: almost half of them are sufficiently confident, whilst $39.5 \%$ of them have a small degree of confidence in their mathematics performance. What is quite relevant from our point of view is that only 9 students feel very confident. The Table 2 shows how many students who have (or don't) a good mathematical background feel confident or not. The majority of students who have a strong background (LS+TE) feel confident at the start of the Maths course, while the majority of "weak" students do not feel confident. As a consequence, it can be argued that the sample of students is quite heterogeneous. That is, the enrolled students are polarized into two clusters that have different degrees of knowledge, acquired skills and attitudes/beliefs towards Maths. The

\footnotetext{
${ }^{4} \mathrm{LS}=$ science and mathematics majors, $\mathrm{TE}=$ applied mathematics, $\mathrm{ART}=$ artistic majors.
} 
following comments provided by two students in Q1 confirm that: one writes that "the pace of the lesson is too fast, even too much", while another student states that "despite the lesson being interactive, the professor is too slow and spent too much time on the easy argument, however some of my colleagues (from weak school) is less confident than me and has difficulties to get into the details".

Table 2. Relation between students' confidence and mathematical background. Strong= Strong mathematical background, Weak= weak mathematical background .

\begin{tabular}{lll}
\hline & Strong & Weak \\
\hline Confident & $65.8 \%$ & $41.4 \%$ \\
Not Confident & $34.2 \%$ & $58.6 \%$ \\
\hline
\end{tabular}

Source: by authors.

\subsection{Research questions}

To recall, this work deals with the design of student-centred activities (SCAs) in LA, more precisely on LTs, a topic that is all but easy to teach and learn due to the intertwining of the syntactic and semantic dimensions.

Hence, our research questions are: RQ1) How can teachers support the learning of LTs? RQ2) To what extent the designed activities may foster a discussion to reveal the emerged difficulties related to LTs?

\section{The student-centred lesson on linear transformations}

In order to answer RQ1, the following lesson about LT was designed and carried out in three sessions, the first two have SCAs whilst the third session does not. In the first session (S1) students have been asked to do some work autonomously during the last hour of tutoring in week 4. They were informed by email, the previous Friday, that the task is composed of three items: i) "study" the video on "Matrices and LTs" (10 mins) on YouTube focusing on the geometrical meanings; ii) explore the applet ${ }^{6}$ "DogCatMouse" according to a set of instructions (see Figure 1), iii) answer a set of questions filling in a table (Figure 2).

\footnotetext{
${ }^{5}$ Video from the YouTube channel 3bluelbrown https://www.youtube.com/watch?v=kYB8IZa5AuE

${ }^{6} \mathrm{https}$ ://demonstrations.wolfram.com/ChangeTheDogMatrixTransformations/
} 


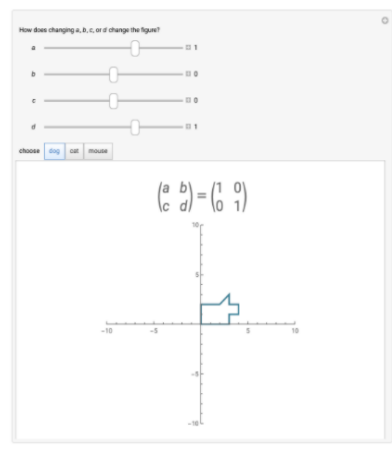

(a)
- open the applet using Chrome

- watch the starting demo carefully

- in the initial configuration, after the demo, the matrix is the identity

- select a figure (Dog/Cat/Mouse).

- change the matrix entries (a, b, c, d), what happens to the pet?

- look at the pet's tail, it does not move from the origin $(0,0)$, why?

(b)

Figure 1. DogCatMouse applet. (a) The preview of the applet designed to explore the geometrical meaning of linear transformations (b) List of instructions designed to guide the exploration of the applet. Source: by authors.

\begin{tabular}{|c|c|c|}
\hline $\begin{array}{l}{[\mathbf{a} b} \\
\mathbf{c} \text { d }\end{array}$ & Descriptions & Entries of the matrix \\
\hline $\begin{array}{ll}{\left[\begin{array}{ll}0 & 0 \\
0 & 1\end{array}\right]}\end{array}$ & & \\
\hline $\begin{array}{ll}{\left[\begin{array}{ll}\mathrm{a} & 0 \\
0 & 0\end{array}\right]}\end{array}$ & the pet shears along the $y$-axis & $a=?$ \\
\hline
\end{tabular}

Figure 2. Sample of the "Fill in table activity": students are asked to fill in the blank in table as part of their work in session 1. Here 2 over 10 rows of the table are reported. Source: by authors.

The second session (S2), a synchronous lesson (90min long), was delivered through a streaming platform ${ }^{7}$ with the support of two digital tools: MS OneNote as virtual board, and Socrative. This session (S2) was composed of three moments: i) ice-break activity, delivered by Socrative, where students should answer five multiple-choice questions concerning the activity in S1 and some properties of the LT; ii) discussion with comments on students' answers to highlight the main properties; iii) cooperative revision of the task assigned in S1, namely commenting students' suggestions on how to fill in the table (Figure 3). This last activity was designed with the purpose of fostering students' awareness of the deep relation between the algebraic and geometrical dimensions of LTs. During the filling in, students formalize the mathematical object guided by the teacher and increase their level of confidence in this topic. The last session (S3) is a tutoring lesson (about 90min long) where the teaching assistant proposes and solves in front of the students a set of standard exercises only indirectly connected to $\mathbf{S 1}$ and $\mathbf{S 2}$.

\footnotetext{
${ }^{7}$ The streaming platform adopted is Cisco Webex Meetings
} 


\section{Data analysis and results}

This section aims at addressing $\mathbf{R Q 2}$ reporting and discussing the data relative to students' answers to the "ice-break" activity during $\mathbf{S 2}$, their answer to the feedback questionnaire (Q3) and their performance on LTs at the midterm exam.

The lesson in $\mathbf{S 2}$ was attended by 86 students, but only 69 participated in the ice-break activity. Their answers confirmed that the majority of them successfully completed the task in S1: indeed $90 \%$ of the students answered correctly to question 1 , in which they were asked about the pet's tail behaviour (see Figure 1b). Moreover, $80 \%$ of them answered correctly to question 5 - "a linear transformation maps straight lines as shown in the figure" - strongly related to a statement and a frame in the recommended video on "Matrices and LTs". The remaining questions were related to the exploration of the applet and the filling-in-table: the answers to question 2 - "if $a=c=1$, and $b=d=0$, the pet does not move. [True/False]" - show that some students $(20 \%)$ have difficulties with the idea and role of the identity matrix, so during the synchronous discussion the focus was on addressing this issue. The question 3 "if $a=c=k>0$, and $b=d=0$, then ..." did not point out any particular difficulties ( $95 \%$ of the students answered correctly), but allowed the instructor to prompt the discussion about the condition " $k>0$ " introducing the topic of reflection transformations. The question 4 - "if a column of the matrix is null, then the pet vanishes. [True/False]" - highlighted an issue about orthogonal projections ( $60 \%$ correct answers). During the discussion the instructor was able to figure out that the mistake had a double origin: on one hand some students did not notice that the pet was "flatten" on the x-axis of the applet, on the other hand some students noticed that, but they thought the pet "had vanished". Such a discussion prompted a discussion also about LT in 3D dimensions, where projections are pivotal for architectural students.

The feedback questionnaire (Q3) to the LT session received only 38 answers. The majority (32 over 38) of the students perceived the three sessions as effective. Almost all the comments confirm that LTs are perceived as a hard topic to learn, even though the SCAs were appreciated. For instance, one student writes: "The topic is new to me, but I like this teaching method, the lessons are not too fast, and the instructors were available to address the issues.", while another student reported that "LT are funnier than calculus" suggesting that the proposed activities do contribute to the goal of making LTs less "out-of-reach".

Finally, in the midterm exam, there were two questions related to LTs: Question 1 was about the properties [2 points], while question 2 asked for the construction of the matrix associated to a given transformation [2 points]. The number of students who attended the midterm exam was 99 , the total average mark for both questions was 1.92 over $4(\mathrm{std}=0.99)$. However, students performed better in question $1(\mathrm{avg}=1.62$, $\mathrm{std}=0.62)$ than in question $2(\mathrm{avg}=0.31$, $\mathrm{std}=0.71$ ). Note that question 1 is more in line with activities performed during in sessions 
S1 and S2, since it requires resorting to properties analysed during these sessions, whilst question 2 is more procedural and in line with session $\mathbf{S 3}$, that didn't include SCAs.

\section{Discussion and conclusions}

The focus of our present research lies on how to support the teaching/learning of LTs (RQ1) and to what extent the designed SCAs may foster a discussion to reveal conceptual emerging difficulties related to LTs (RQ2). In particular, to check if the use of OERs, such as videos and applets, does foster a deep understanding of the dual nature of LTs. The results of our investigation reveal that, during the three sessions, the proposed SCAs have prompted students to participate actively, mainly in S2, providing comments and making questions, unfolding conceptual difficulties and misconceptions on the dual nature of LTs. More precisely, the asynchronous session (S1) was effective both in fostering students' thinking and in sharing an instrument that both the teacher and the students can use during synchronous sessions (Andrà et al., 2019). The use of OERs paired with SCAs sustained therefore the students' deeper understanding of a challenging topic, reducing the "out-ofreach" feeling towards Maths and LA (Stewart, 2017) and fostering the connection between syntactic and semantic dimensions (Lalaude-Labayle et al., 2018). Nevertheless, this preliminary study has its limitations, the major ones concern the data collections: the anonymous nature that does not allow always to link answers in different datasets; the cardinality of the sample that changes along the weeks and does not allow to have the whole picture but just a glance. Despite those limitations, which are going to be addressed in a future study, the current findings allow us to claim that a similar approach can be used to approach other mathematical concepts, with appropriate adjustments, to implement active learning strategies among STEAM students.

\section{References}

Andrà, C., Bernardi, G., \& Brunetto, D. (2019). Teaching with emerging technologies in a STEM university math class. In 5th International Conference on Higher Education Advances (HEAd'19) (pp. 963-971). 2019, Editorial Universitat Politècnica de València.

Felder, R. M., Brent, R. (2009). Active learning: An introduction. ASQ Higher Education Brief, 2(4), 1-5.

Felder, R. M., Felder, G. N., Dietz, E. J. (1997). A longitudinal study of alternative approaches to engineering education: survey of assessment results. In Proceedings Frontiers in Education 1997 27th Annual Conference. Teaching and Learning in an Era of Change (Vol. 3, pp. 1284-1289). IEEE.

Gueudet, G. (2008). Investigating the secondary-tertiary transition. Educational studies in mathematics, 67(3), 237-254. 
Lalaude-Labayle, M., Gibel, P., Bloch, I., and Lévi, L. (2018, April). A TDS analytical framework to study students' mathematical activity. An example: linear transformations at University. In Proceedings of INDRUM 2018, Second conference of the International Network for Didactic Research in University Mathematics. 234-243.

Lay, D., Lay, S., McDonald, J. (2016). Linear Algebra and Its Applications. Pearson Ed. Limited.

Moura Santos, A., Costa, L. (2020) Linear Algebra Flipped Classroom with a MOOC, submitted to Interactive Learning Environments.

Prince, M. (2004). Does active learning work? A review of the research. Journal of Engineering Education, 93(3), 223-231.

Santiago, P., Donaldson, G., Herman, J., Shewbridge, C. (2012). OECD Reviews of Evaluation and Assessment in Education: Portugal 2012, OECD Publishing, Paris, http://dx.doi.org/10.1787/9789264117020-en.

Stewart, S. (2017). School Algebra to Linear Algebra: Advancing Through the Worlds of Mathematical Thinking. In: Stewart, S. (Ed.), And the Rest is Just Algebra (pp. 219-233). Springer. 